\title{
Electro-Optical Properties of Liquid Crystals Composite with Zinc Oxide Nanoparticles
}

\author{
H. ESKALEN ${ }^{a}, *$ S. ÖZĞ AN $^{b}, \ddot{U} \cdot \operatorname{ALVER}^{c}$ AND S. KERLİ ${ }^{d}$ \\ ${ }^{a}$ Material Science and Engineering Program, Graduate School of Natural and Applied Science, \\ Kahramanmaras Sutcu Imam University, Kahramanmaras, Turkey \\ ${ }^{b}$ Department of Physics, Kahramanmaras Sutcu Imam University (KSU), Kahramanmaras, Turkey \\ ${ }^{c}$ Department of Metallurgical and Materials Engineering, Karadeniz Technical University, Trabzon, Turkey \\ ${ }^{d}$ Department of Energy Systems Engineering, Faculty of Elbistan Technology, KSU, Kahramanmaras, Turkey \\ (Received May 1, 2013; in final form October 13, 2014) \\ In this study, zinc oxide $(\mathrm{ZnO})$ nanoparticles were synthesized by spray pyrolysis method. The properties of \\ $\mathrm{ZnO}$ nanoparticles are determined by means of scanning electron microscope (SEM), X-ray diffraction (XRD) and \\ UV-vis spectroscopy. Nematic liquid crystal mixture E7 was doped with 1, 2, and $4 \% \mathrm{ZnO}$. We investigate electrical \\ and optical parameters of pure and doped nematic liquid crystal mixture E7. The experiments related to voltage \\ dependence and light transmittance of the pure and doped E7 were carried out. The values of threshold voltage, \\ total phase retardation and birefringence are examined. The results show that doping zinc oxide nanoparticles into \\ liquid crystal mixture E7 decreases threshold voltage significantly.
}

DOI: 10.12693 /APhysPolA.127.756

PACS: 78.20.Jq, 77.84.Nh, 78.20.Fm

\section{Introduction}

Organic materials have been increasingly examined over the past three decades due to their potential application areas and interesting properties. Specifically, liquid crystals (LCs), as a fourth state of matter, are used for different electro-optic devices. Liquid crystal displays technology plays a key role at the notebook, computer displays and flat TV, because of showing full range of color, lower power consumption and using lower space [1]. In 2006, annual sales of LCs was more than 100 billion at the industrial applications [2]. They are also used at the different areas such as; electro-optic filters [3], holography $[4,5]$, digital data storage [6-8], and biosensors [9].

Generally, wide temperature range of the LCs phase, high optical and dielectric anisotropy and fast switching time are required for modern LCs industrial applications. Synthesizing new LCs or composing liquid crystals mixture [10] and using guest materials are two basic methods for obtaining LCs with these properties, thus performance of the device that uses liquid crystals strongly depends on LC and guest materials [11, 12].

Dye, polymer and nanoparticles are main types of guest materials. The $1 \%$ dye doping in nematic liquid crystals increases reorientation of the nematic liquid crystal (NLC) up to twice [13] and it was found that doping dye into nematic liquid crystals decreases threshold voltage by almost $25 \%$ and threefold the refractive index modulation [14]. Moreover, critical frequency decreases with doping Disperse Red 1 dye to NLC [15].

*corresponding author; e-mail: heskalen@gmail.com
Combination of polymer doped liquid crystals (PDLC) and holography create a new material holographic PDLC (H-PDLC) and these new materials have been used in many new applications [16]. The last guest material for enhancement of the performance of LCs devices is nanomaterials. Doping nanomaterial in LCs enhances the properties of LCs. In literature, different type of nanomaterials are used for this purpose such as metallic nanoparticles [17], ferromagnetic nanoparticles [18], semiconducting nanoparticles [19] and carbon nanomaterials $[1,20]$.

Due to the simple production, having large band gap energy $3.37 \mathrm{eV}$ and high exciton binding energy $60 \mathrm{meV}$, $\mathrm{ZnO}$ nanoparticles (NPs) are used in lot of application areas from electronics to optics; solar cells to gas sensors [21]. In 2009, it was found that doping zinc oxide NPs to nematic liquid crystals considerably decreased threshold voltage and this may help the lower energy consumption of liquid crystals devices [22]. Moreover, zinc oxide NPs increased the temperature range of the LCs phase [23] and enhanced orientation of liquid crystals [24]. In this study, $\mathrm{ZnO}$ NPs were synthesized by a simple spray pyrolysis method. The properties of $\mathrm{ZnO}$ NPs are determined by means of scanning electron microscopy (SEM), X-ray diffraction (XRD) and UV-vis spectroscopy and then nematic liquid crystal mixture E7 was doped with $0,1,2$, and $4 \% \mathrm{ZnO}$ NPs. Electrical and optical properties of these samples were investigated.

\section{Experimental detail}

\subsection{Material}

The liquid crystal used in this experiment was the commercial eutectic nematic mixture E7 (obtained from Synthon Chemicals) that has a positive dielectric anisotropy 
given in Fig. 1. LC cells were on indium-thin oxide (ITO) coated glass substrate with planar alignment layer and the thicknesses of cells were about $8 \mu \mathrm{m}$ controlled by spacer. The cells were purchased from Instec, Inc.

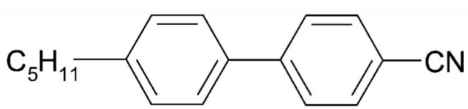

$25 \%$

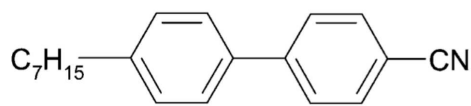

$16 \%$

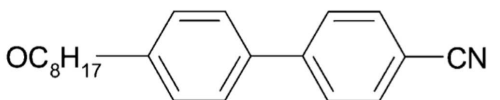

$8 \%$

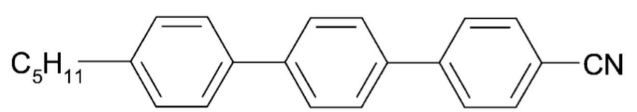

Fig. 1. Chemical composition of the nematic liquid crystals mixture E7.

\subsection{Method}

$\mathrm{ZnO}$ NPs were synthesized by spray-pyrolysis methods. The production schema of zinc oxide NPs is shown in Fig. 2. Zinc nitride $\left(\mathrm{Zn}\left(\mathrm{NO}_{3}\right)_{2} \cdot 6 \mathrm{H}_{2} \mathrm{O}\right)$ salt was used to prepare NPs. $0.5 \mathrm{M}$ solutions were formed by using $100 \mathrm{ml}$ pure water. The solution evaporated with the aid of ultrasonic nebulizer and passed through tube furnace having $600{ }^{\circ} \mathrm{C}$ temperature and then evaporated solution collected to the inner side of container whose outer side was covered with water. The obtained NPs were inverted to powder by help of centrifuge and then stored for analysis. LC and ZnO NPs were dissolved in chloroform, and then they were mixed together for several hours until they became homogeneous, using an ultrasonic bath at $65^{\circ} \mathrm{C}$ and a mechanical stirrer at room temperature. The mixture was left for one night in order to fully evaporate chloroform. Afterwards, 0, 1, 2, and $4 \mathrm{wt} \%$ ratio of $\mathrm{ZnO} \mathrm{NPs}$ were used to prepare NLC mixtures. Empty cells were filled with the NPs doped NLC mixture by capillary action at the $10^{\circ} \mathrm{C}$ above the $\mathrm{LC}$ clearing point. The edges of the samples were sealed by epoxy. The voltage dependence of dielectric constant of these materials were measured by using a HP 4194A impedance analyzer with the applied voltage range $0-40 \mathrm{~V}$ at room temperature.

Voltage dependent optical transmittance experiment was performed by laser, polarizer analyzer and photodiode. The experimental schema of experiment is given in Fig. 3. The frequency of voltage adjusted to $500 \mathrm{~Hz}$ and wavelength of laser was $514 \mathrm{~nm}$. The difference between ordinary and extraordinary ray is called birefringence

$$
\Delta n=n_{\|}-n_{\perp},
$$

where $n_{\|}$is the refractive index measured parallel to director and $n_{\perp}$ is the refractive index measured perpendicular to director. For electro-optic devices birefringence is related to light transmission though LCs cell,

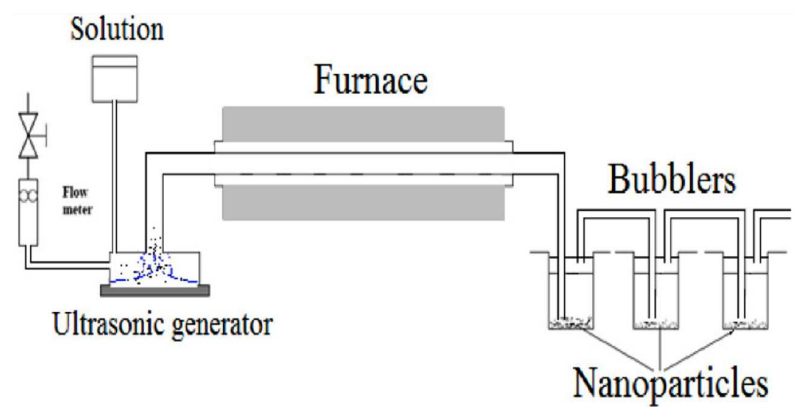

Fig. 2. Experimental setup for spray pyrolysis method.

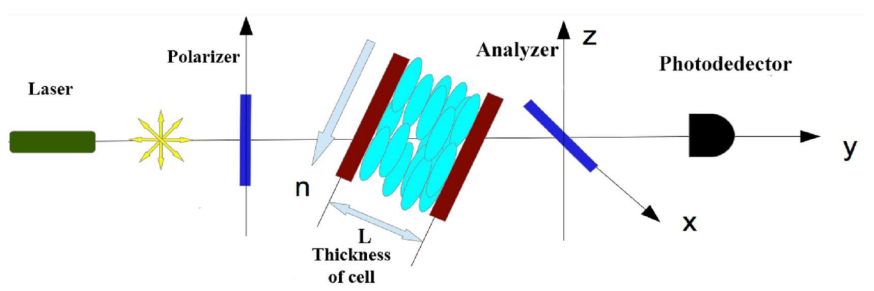

Fig. 3. Experimental setup for optical measurements.

$$
I=I_{0} \sin ^{2} 2 \Psi(1-\cos \delta \Phi) / 2,
$$

where $I$ is the transmitted intensity of light, $I_{0}$ is the initial intensity of the linearly polarized beam, $\Psi$ is the angle between polarizer and analyzer and is adjusted to $45^{\circ}$. $\lambda$ is the wavelength of laser and $L$ is the thickness of the cell. $\delta \Phi$ is the total phase difference and it is related to the birefringence such that

$$
\delta \Phi=(2 \pi / \lambda) \Delta n L .
$$

To compute the retardation $\Phi_{V}$ at any voltage $V$, Eq. (4) is used [25]. In this equation $I$ is the intensity of given voltage, $I_{\max }$ is the maximum intensity and $n$ is the number of oscillation. As the magnitude of applied voltage increases, the number of $n$ decreases i.e. for

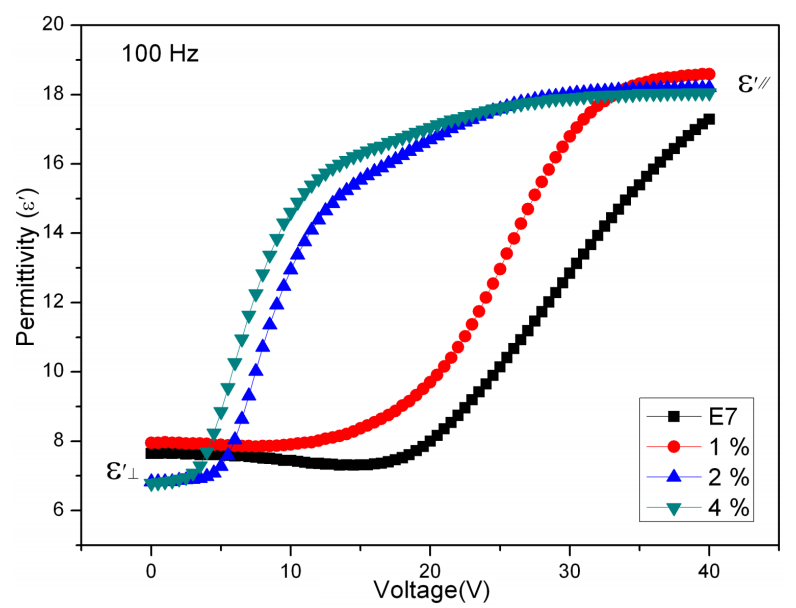

Fig. 4. Variation of dielectric constant with the bias voltage. 
$2.3<V<8, n$ has a value of 0 , for $1.4<V<2.3, n$ has value of 1 , for $0.9<V<1.4, n$ has a value of 2 and $0<V<0.9, n$ has a value of 3 . In pure E7, for example, $I$ is 3650 a.u. at zero voltage, $I_{\max }$ is 4430 a.u. and $n$ is 3 . The retardation at zero voltage, $\Phi_{0}$, is $21.124 \mathrm{rad}$. If the wavelength of laser and thickness of LCs cell is known, then the birefringence of E7 at zero voltage can be calculated by help of Eq. (3). If one changes thickness of cell by an amount of $0.1 \times 10^{-6}$ then the birefrigence of system changes about $3.5 \times 10^{-3}$ i.e. a small change in thickness affects birefrigence of system largely.

$$
\Phi=\cos ^{-1}\left(1-\frac{2 I}{I \max }\right)+2 n \pi
$$

One of the most important physical properties of the liquid crystalline compounds is dielectric anisotropy $(\Delta \varepsilon)$. Dielectric anisotropy is described as $\Delta \varepsilon=\varepsilon_{\|}-\varepsilon_{\perp}$ where $\varepsilon_{\|}$and $\varepsilon_{\perp}$ are parallel and perpendicular permittivities to the director of liquid crystals. Perpendicular part of dielectric constant versus voltage is given in Fig. 4, and dielectric anisotropy $\Delta \varepsilon$ values were measured at $100 \mathrm{~Hz}$ frequency. Real part of dielectric permittivity $\varepsilon^{\prime}$ was defined as:

$$
\varepsilon^{\prime}=\frac{C d}{\varepsilon_{0} A} \text {. }
$$

Here $C, d, \varepsilon_{0}, A$ are capacitance, cell thinkness, the permittivity of free space $\left(\varepsilon_{0}=8.85 \times 10^{-14} \mathrm{~F} / \mathrm{cm}\right)$ and actice cell area, respectively.

\section{Results and discussion}

The XRD pattern of $\mathrm{ZnO}$ nanoparticles were synthesized by spray-pyrolysis methods given in Fig. 5 . The diffraction peaks show that the produced particles have hexagonal phase with wurtzite structure consistent with the values in the standard card (PDF-2, reference code: 00-036-1451). The SEM of ZnO NPs produced by spray-pyrolysis methods is shown in Fig. 6. As shown in Fig. 6, ZnO NPs have a shape with hollow spheres. The absorption spectrum of $\mathrm{ZnO}$ NPs dispersed in pure water is given in Fig. 7. The absorption edge at $375 \mathrm{~nm}$ matches to $\mathrm{ZnO}$ phase. This edge implies that $\mathrm{ZnO}$ NPs have a band gap energy with $3.3 \mathrm{eV}$.

Figure 4 clearly shows that at lower voltage dielectric constant does not change, however, after using a certain voltage, which is defined as threshold voltage, the real part of dielectric constant increases sharply. Alignment of ITO cell is planar, at zero voltage, by applying electrical field to ITO cell, the planar alignment of liquid crystals starts to change and align in the direction of electrical field, that is, homogeneously aligned liquid crystals switch to heterogeneous alignment. Threshold voltage is determined by $10 \%$ increment of the initial dielectric constant and threshold voltage decreases from 20 to 16.5 at the $1 \%, 5.4$ at the $2 \%$ and 3.8 at the $4 \%$ zinc oxide NPs concentration, respectively. As indicated in Fig. 4, doping E7 with $\mathrm{ZnO}$ NPs decreases threshold voltage. For $1 \%$ doped sample, threshold voltage decreases by a small amount but for $2 \%$ doped sample threshold voltage decreases significantly. For $4 \%$ amount of increase,

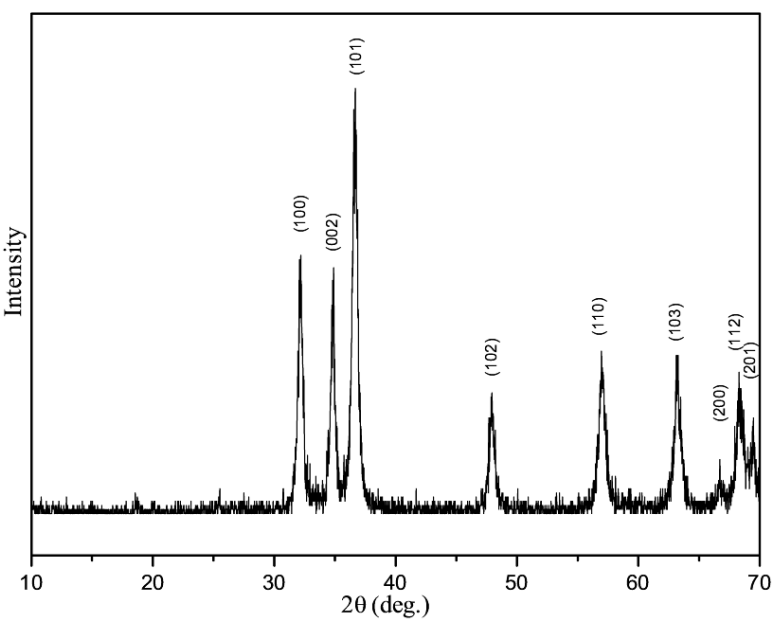

Fig. 5. X-ray diffraction pattern of ZnO NPs.

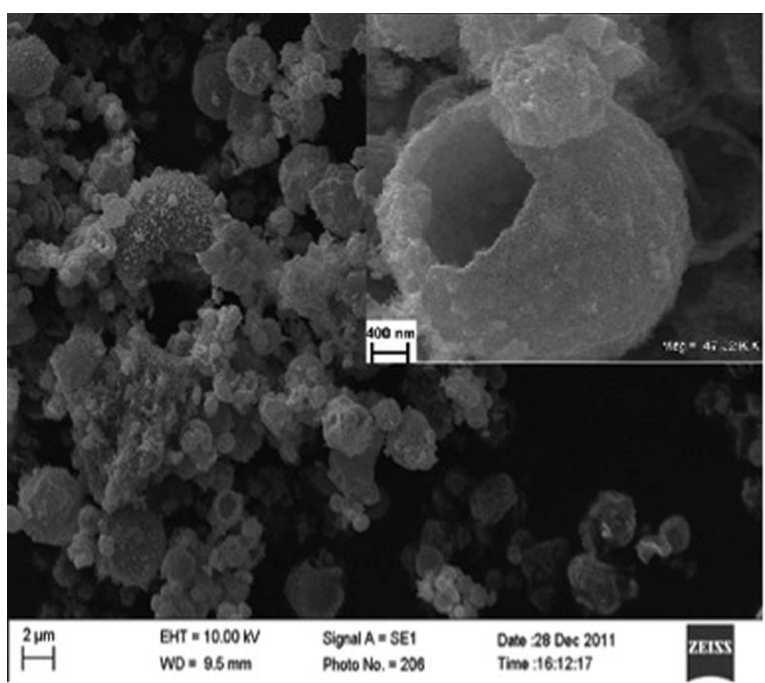

Fig. 6. The SEM images of $\mathrm{ZnO}$ NPs.

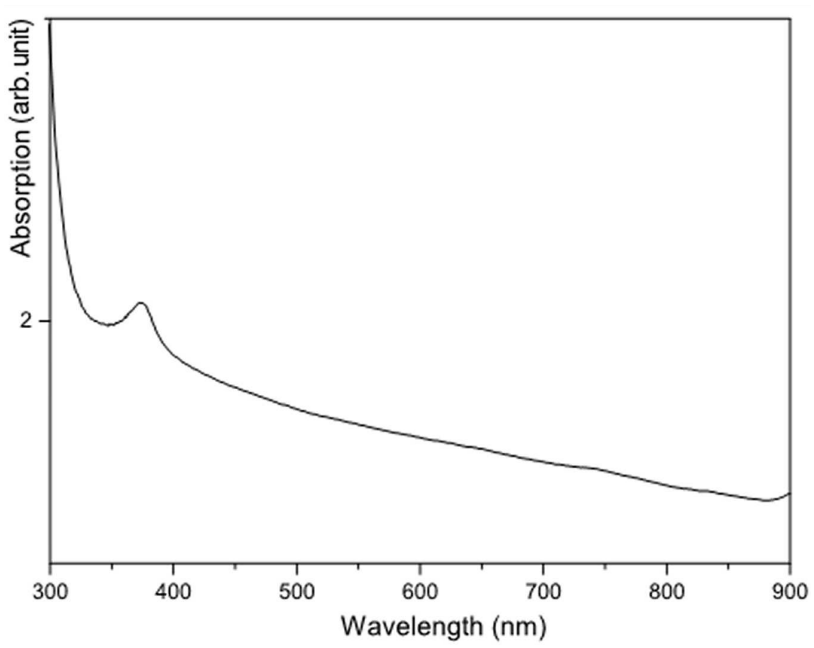

Fig. 7. UV-vis spectra of ZnO NPs. 
it decreased again. Slopes of samples in Fig. 4 imply how fast liquid crystals and nanomaterial change their direction to the direction of applied electrical field; slopes are also frequency dependent, by changing frequencies lead to obtain different slopes.

Figure 3 depicts the experimental setup for voltage dependence of optical transmittance. Transmittance of pure and doped liquid crystals are given in Fig. 8A-D. The values of phase retardations at any voltage could be calculated by using Eq. (4) and birefringence can be found by Eq. (3). In Fig. 8 while the voltage increases the light transmittance starts to alter as maxima and minima peaks correspond to an odd or an even integer of Eq. (2). When the applied voltage increases more, it is observed that the birefringence values approach to zero. As seen from Fig. 8A-D, pure E7 and zinc oxide NP doped liquid crystals mixtures have almost the same birefringence values at the zero voltage. In addition as shown from Fig. 8A-D, doping $\mathrm{ZnO}$ NP changes the retardation and birefringence of $\mathrm{E} 7$ at higher voltage.
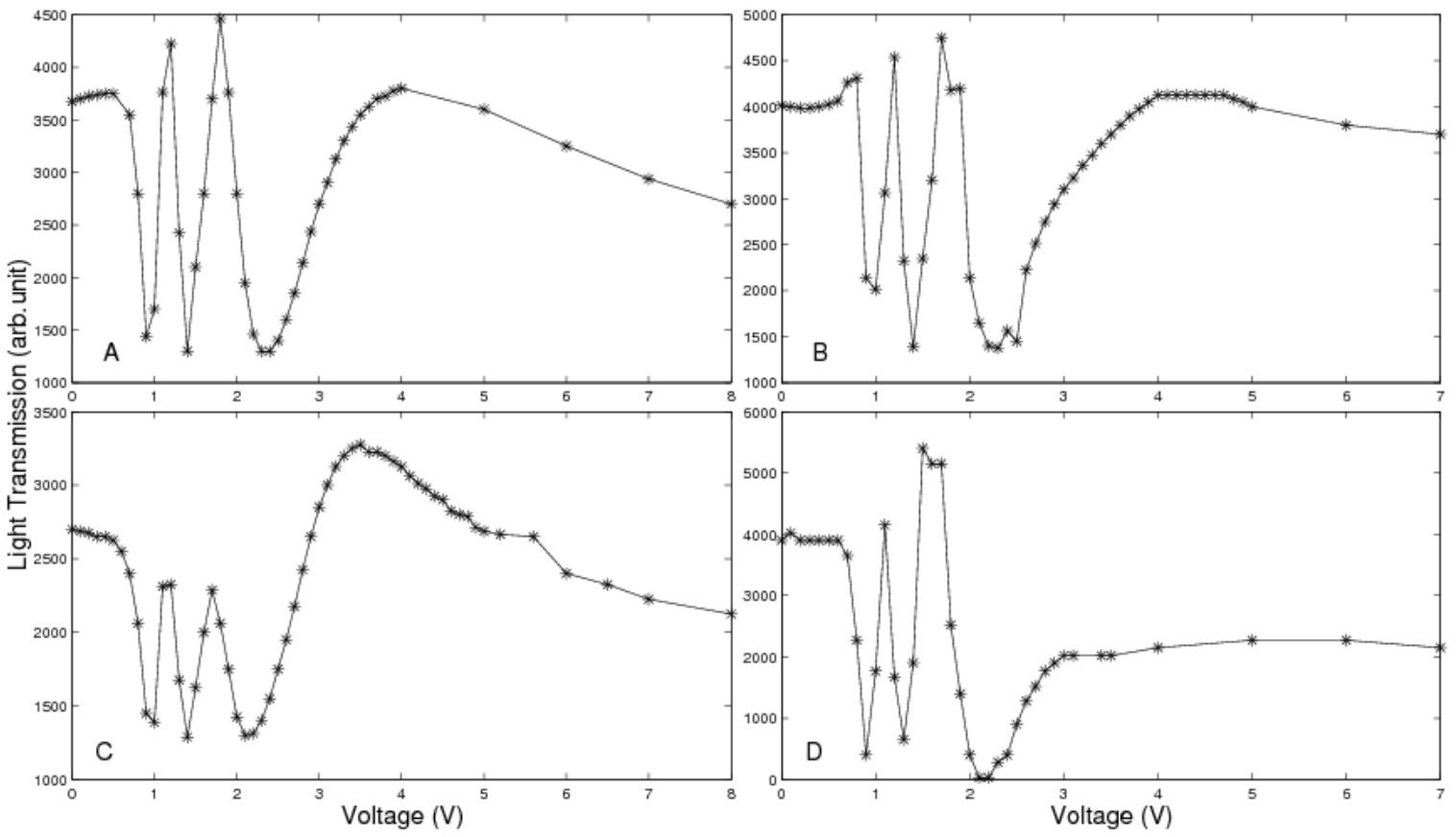

Fig. 8. Dependence of optical transmittance of E7 1, 2, and $4 \%$ on voltage: (A) optical transmittance of pure E7 on voltage, (B) optical transmittance of $1 \%$ zinc oxide doped E7 on voltage, (C) optical transmittance of $2 \%$ zinc oxide doped E7 on voltage, (D) optical transmittance of $4 \%$ zinc oxide doped E7 on voltage.

These results indicate that $\mathrm{ZnO}$ NPs decrease threshold voltage. This means that electro-optical characteristics of NLC mixture E7 is enhanced by ZnO NPs. This might be explained by that while creating LC cells, static electricity might be generated, and then purity ions in LC mixed together; as a result of this process, impurity ions were created. Normally, impurity ions moderately affect the threshold voltage and response time of NLC cells [26, 27], but zinc oxide NPs in NLC might capture impurity ions and affect the electro-optical properties significantly. This is referred to field screening effect (FSE). Moreover, since ZnO NPs are larger in size than NLC, ZnO NPs dipole moments are higher than NLC, this causes higher electrical torque of $\mathrm{ZnO}$ NPs than of NLC [27, 28]. When electric field is applied to LCs cell, ZnO NPs with high electrical torque also positively affect anchoring condition. These two reasons might cause an enhancement of electro-optical properties of zinc oxide dispersed in NLC mixtures, however, the exact reason of decreasing threshold voltage is still unclear. Further studies are needed to understand exact mechanism.

\section{Conclusion}

In this study, we synthesized zinc oxide NPs by spraypyrolysis method and then they were doped into nematic liquid crystal mixture E7. The applied voltage dependence of the dielectric properties of pure and $\mathrm{ZnO}$ doped mixtures have been examined at room temperature. Phase retardations and birefringence at given voltage could be derivated from optical transmittance experiment and related equations. It is clearly seen that threshold voltage decreased dramatically with addition of zinc oxide NPs to E7. We assume that electro-optical properties of liquid crystals can be changed by $\mathrm{ZnO}$ doping concentration. Moreover power consumption obtained by LC with lower threshold voltage is less, when it is concerned in industrial application. 


\section{Acknowledgments}

The work has been supported by Kahramanmaras Sutcu Imam University Scientific Research Projects Coordination Department under Project No. 2012/5-13 M.

\section{References}

[1] W. Lee, J.-S. Gau, H.-Y. Chen, Appl. Phys. B 81, 171 (2005).

[2] J.A. Castellano, Am. Scient. 94, 438 (2006).

[3] S.-T. Wu, Appl. Opt. 28, 48 (1989).

[4] A.G. Chen, D.J. Brady, Opt. Lett. 17, 441 (1992).

[5] M. Eich, J.H. Wendorff, Makromol. Chem., Rapid Commun. 8, 467 (1987).

[6] I.C. Khoo, M. Shih, M.V. Wood, B.D. Guenther, P.H. Chen, F. Simoni, S.S. Slussarenko, O. Francescangeli, L. Lucchetti, Proc. IEEE 87, 1897 (1999).

[7] M. Eich, J.H. Wendorff, B. Reck, H. Ringsdorf, Makromol. Chem., Rapid Commun. 8, 59 (1987).

[8] A.S. Matharu, S. Jeeva, P.S. Ramanujam, Chem. Soc. Rev. 36, 1868 (2007).

[9] S.J. Woltman, G.D. Jay, G.P. Crawford, Nature Mater. 6, 929 (2007).

[10] Ş. Özğan, M. Okumuş, Braz. J. Phys. 41, 118 (2011).

[11] L. Dolgov, O. Yaroshchuk, M. Lebovka, Mol. Cryst. Liq. Cryst. 496, 212 (2008).

[12] K.K. Vardanyan, E.D. Palazzo, R.D. Walton, Liq. Cryst. 38, 709 (2011).

[13] I. Janossy, Phys. Rev. E 49, 2957 (1994).
[14] O. Koksal, M. Okutan, M. Gokcen, Opt. Commun. 284, 4924 (2011).

[15] M. Okutan, O. Köysal, S.E. San, Y. Köysal, ISRN Nanomaterials, 596125 (2012).

[16] Y.J. Liu, X.W. Sun, Adv. OptoElectron., 684349 (2011).

[17] S. Kobayashi,T. Miyama, N. Nishida, Y. Sakai, H. Shiraki, Y. Shiraishi, N. Toshima, J. Display Technol. 2, 121 (2006).

[18] F. Brochard, P.G. de Gennes, J. Phys. 31, 691 (1970).

[19] T. Zang, C. Zhong, J. Xu, Jpn. J. Appl. Phys. 48, 055002 (2009).

[20] W. Lee, C-S. Chiu, Opt. Lett. 26, 521 (2001).

[21] H. Li, Y. Zhang, H. Liu, J. Wang, J. Am. Ceram. Soc. 94, 3267 (2011).

[22] H. Jiang, N. Toshima, Chem. Lett. 38, 566 (2009).

[23] A. Malik, A. Choudhary, P. Silotia, A.M. Biradar, J. Appl. Phys. 110, 064111 (2011).

[24] L.J. Martinez-Miranda, K.M. Traister,I. MeléndezRodríguez, L. Salamanca-Riba, Appl. Phys. Lett. 97, 223301 (2010).

[25] M.B. Jalil, in: Education and Training in Optics and Photonics, Optical Society of America, Tucson (AR) 2003

[26] K. Neyts, S. Vermael, C. Desimpel, J. Appl. Phys. 94, 3891 (2003).

[27] H.J. Kim , Y.G. Kang, H.G. Park, K.M. Lee, S. Yang, H.Y. Jung, D.S. Seo, Liquid Crystals 38, 871 (2011).

[28] H.Y. Jung, H.J. Kim, S. Yang, Y.G. Kang, B.Y. Oh, H.G. Park, D.S. Seo, Liq. Cryst. 39, 789 (2012). 